\title{
Integrated design of micro force sensor oriented to cell micro-operation
}

\author{
Zhen An, Chaoqun Xiang, Jinming Wang, Liping Dong and Lina Hao* \\ College of Mechanical Engineering and Automation, Northeastern University, Shenyang, Liaoning, \\ China
}

\begin{abstract}
.
BACKGROUND: During the process of cell micro operation, the contact force between the entry needle and the cell membrane is usually a very small area to be tested.

OBJECTIVE: These two features have been utilized to design a high precision micro force sensor to provide a force feedback in the process of cell micro operation.

METHODS: However, PVDF (Polyvinylidene Fluoride) has continued to display both a wide range of frequency response and a highly sensitive transform between force and electricity. In this paper a charge signal is obtained. In order to transform the charge signal into a voltage signal, a signal processing circuit was designed. The data acquisition card PCI-6221 (National Instruments) was used. It was employed to collect the voltage signal.

RESULTS: The resultant micro force sensor was calibrated by using a rating test.

CONCLUSIONS: The experimental test results demonstrated that the sensitivity and linearity of the designed micro force sensor were higher than the sensitivity and linearity of a traditional piezoelectric sensor.
\end{abstract}

Keywords: Cell micro-operation, micro force sensor, PVDF, charge amplifier

\section{Background}

The rapid development of bioengineering, cell engineering, genetic engineering and cell injection as frontier technologies has made it possible to carry out advancements in gene therapy. In the field of genetic engineering and biomedicine, a manipulator is usually needed in order to operate on a single cell. In the process of cell injection, when the entry needle does not contact the cell, the needle can then be guided by vision. But the vision approach cannot tell whether the entry needle and the cell actually contacted each other and whether or not the needle actually threaded the cell membrane. In [1], the process of cell micro operation ensures that the force of the entry needle directly influences the survival rate of the cell. Traditionally, this would entirely rely on the experience of the operator. On average, it would cost more than half a year to train and develop required operator skills. This is because the operator training process would also be affected by the mental factors of the operator. Today, in order to realize automation, generalization, and ease of operation in cell micro operation, highly sensitive micro force sensors are required. These would precisely detect the injection processes for cells as required.

\footnotetext{
${ }^{*}$ Corresponding author: Lina Hao, College of Mechanical Engineering and Automation, 319\#, Northeastern University, Shenyang, Liaoning, China. Tel.: +86 0248368 3010; E-mail: haolina@me.neu.edu.cn. 


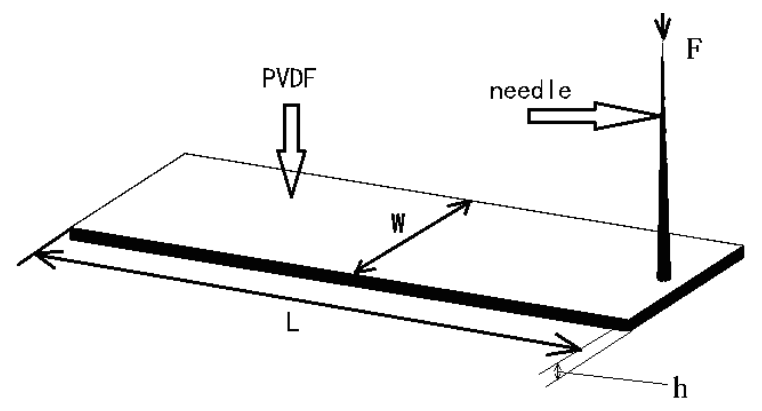

Fig. 1. The structure picture of PVDF.

The PVDF micro force sensor designed and presented in this paper can achieve this precision. It can be applied in the field of gene therapy, as described in [2-4].

There are two theories that can be used explain the measure of micro force. The first one uses a resistance variation transducer. In [5-7], Fung and Shen et al., designed a one dimension and later a two dimension cantilever beam micro force sensor that was based on PVDF. However, the sensor could only measure the dynamic force. In [8], Brunetto et al., utilized a smart material IPMC to design dynamitic sensor to measure the viscosity of liquids. Because generally, the stability of IPMC is not very good, the sensor could only measure dynamic force. Given the limitations encountered in the above research works, a smart piezoelectric material PVDF is used in this paper in order to bring into existence a specific design for a highly sensitive micro force sensor.

\section{The model of PVDF piezoelectric sensor's physical system}

For many decades now, research on piezoelectric materials has not been continued to take place. In 1969, Kawai discovered a high polymer material known, Polyvinylidene Fluoride (abbreviation PVDF). We note that PVDF has a very high piezoelectric effect. In the same year, some Americans researchers discovered piezoelectricity in polyvinyl chloride (abbreviated PVC). They also researched on the methods used to enhance the activity of PVDF. In [9], acoustic equipment underwater, pressure sensor and piezoelectric lighter were designed. But continued research on PVDF has indicated that the material does not only have a large dielectric constant, better piezoelectric and pyro-electric properties, but that it also has very excellent mechanical properties. In [10], the material attracted extensive research attention when it was introduced into the scene. PVDF has continued to emerge as the most ideal sensing material. As will be seen in this paper, in the process of micro operation, a micro force sensor that is based on PVDF's intelligent smart material will always be the most practical implementation. In this design we also use a cantilever beam. This is because since the cantilever beam can detect very tiny forces and pressures, its structure is the one that is usually used in the design of micro force sensors. Figure 1 presents the structure of PVDF.

$W$ is the width and $L$ is the length of the PVDF film. $h$ is its thickness and $A$ is its superficial area given as $(L \times W)$. $a$ is the cross sectional area given as $(W \times h)$ and $d_{31}$ is the piezoelectric constant of PVDF. According to piezoelectric property, the charge generated on the surface of PVDF can be calculated by the follow formula

$$
Q=\int d_{31} \delta d A=\iint d_{31} \delta d z d x
$$




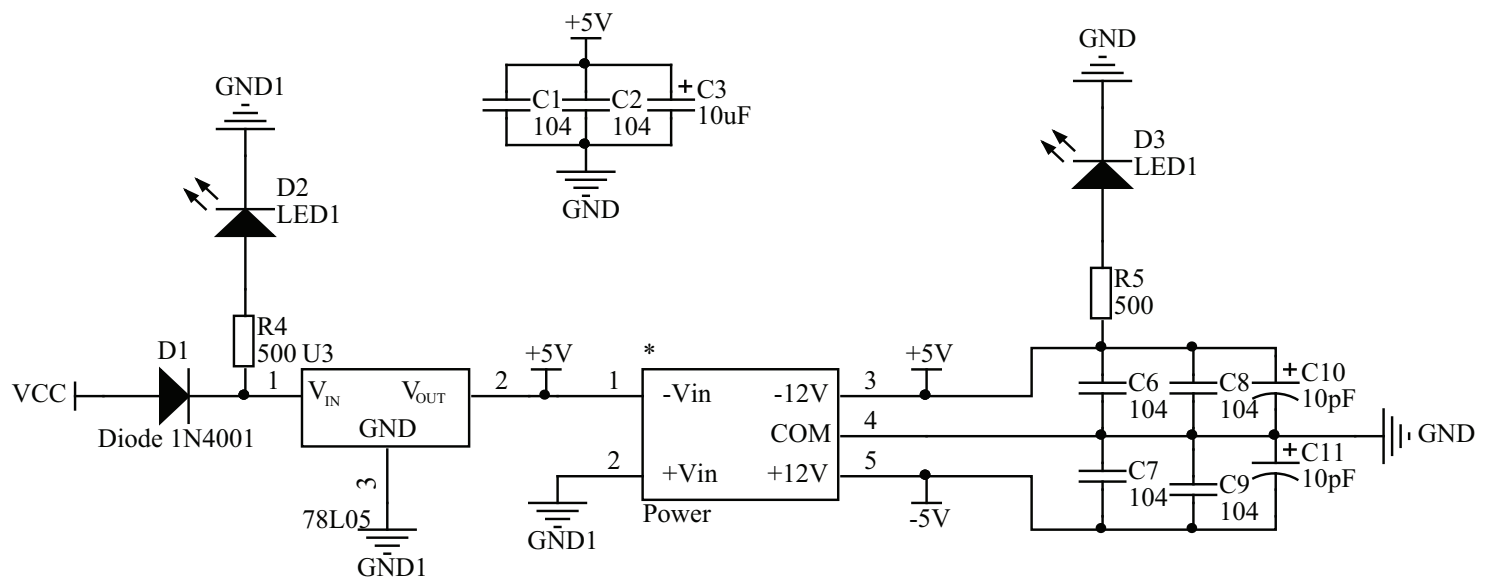

Fig. 2. Power circuit.

where, $d_{31}$ is the piezoelectric coefficient. $\delta$ stands for the unit force along the length direction, $L$. Thus

$$
\delta=\frac{F L \frac{h}{2}}{I_{Z}}
$$

$F$ stands for the force acting on the tip, and $I_{z}$ is the moment of inertia along the $z$ direction.

Thus

$$
\begin{aligned}
& I_{z}=\frac{w h^{3}}{12} \\
& Q=\frac{3 d_{31} L^{2}}{h^{2}}
\end{aligned}
$$

The above formulae show the relationship between the force on the PVDF surface and the charge generated on the surface. Note that in practical applications, it is quite hard to detect the charge. Hence, in order to conveniently measure the signal, reduce noise and enhance the accuracy of micro force sensor, it is necessary to design a signal processing circuit to transform the charge signal into a voltage signal.

\section{The integrated design}

\subsection{The design of the hardware circuit}

PVDF signal disposal circuit consists of a power supply, electric charge amplifier circuit and a voltage amplification circuit. Power supply circuit generates a voltage of $\pm 12 \mathrm{~V}$ required to supply the rest of the conditioning circuit. The power supply module is designed to be small but it gives very high efficiency and can easily convert voltage polarity between positive and negative. It supplies power both for the IC used in the charge amplification circuit and the IC used in the voltage amplification circuit. The LEDs D1 and D2 are used as power-on indicators as shown in Fig. 2.

The charge amplifier circuit is an integral operation circuit which can convert charge into voltage. In Fig. 3(a), the capacitive characteristic of PVDF implies stored charge. This can be considered equivalent 


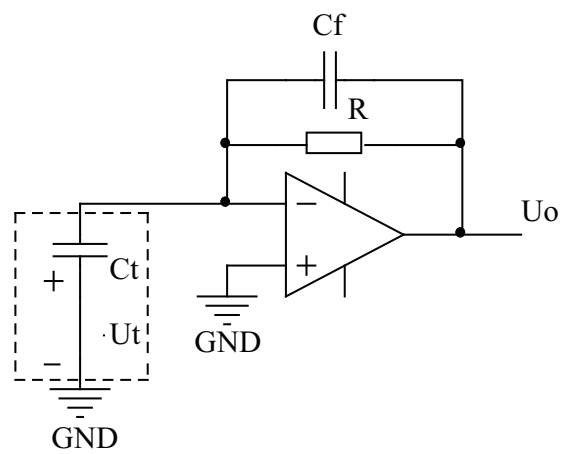

(a) charge amplifier circuit schematics

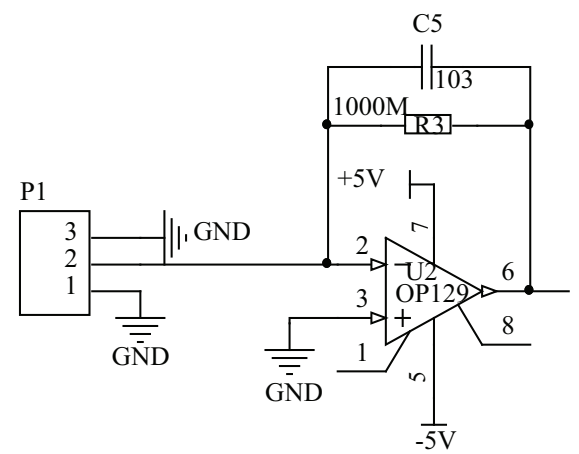

(b) charge amplifier designed in the article

Fig. 3. Charge amplifier schematic.

to the voltage. The voltage $U_{t}$ cascades with an output capacitance in the imaginary line frame of the Fig. 3(a). The relationship of $U_{t}, C_{f}$ and the charge of the capacitance are as follow

$$
U_{t}=\frac{Q}{C_{f}}
$$

In this research work, as shown in the Fig. 3(b), the IC of charge amplifier adopts OPA129. OPA129 is an ultralow bias current differential operational amplifier. It can restrain common-mode interference signals quite sufficiently. Usually, resistance $R$ is parallel connected with $C_{f}$ in order to prevent capacitor $C_{f}$ from being charged for a long time. Excessive charging of $C_{f}$ can lead to saturation of the integrated opamp. Then, in case $\frac{1}{\omega C_{f}} \ll R$, the output signal frequency of the sensor cannot be too low. So $f$ must be greater than $\frac{1}{2 \pi R C_{f}}$. In order to design quasi electric charge amplifier, therefore, the operating frequency of charge amplifier must be lower than $10^{-3} \mathrm{~Hz}$. In order to accurately realize the static measurement and calibration of piezoelectric sensor, the key technology of the charge conversion unit should have static properties. By the amplifying circuit for electric charge, the perceptive charge that is generated by the stress of the PVDF is converted to voltage signal. The voltage that is generated by the charge amplifying circuit is in the $\mathrm{mV}$ range. Hence, the voltage signal needs to be amplified again through a voltage amplifier circuit. In Fig. 4, the IC used for the voltage amplifier is AD620. It is a low cost, high precision instrumentation amplifier. This IC is applied with reasonable simplicity. Only an external resistor is used to set its gain. At the end, the voltage signal generated by the signal processing circuit was collected through a Data Acquisition Card.

\subsection{Structure design}

The structure representation of one dimension micro force sensor based on PVDF is shown in Fig. 1. A cantilever beam structure was adopted on the structure. A needle was installed on the terminal of the PVDF. Meanwhile, in order to increase rigidity of the PVDF membrane, two pieces of plastic film were used to pack the PVDF membrane. Two electrodes were extracted from the inside of the metal box. The metal box was designed to avoid EMI (Electromagnetic Interference) within its environment. A coaxial signal cable was used as a signal line. It was also ensured that the cable would avoid EMI within its environment. All of these were intended to increase the stability of the dsigned micro force sensor. 


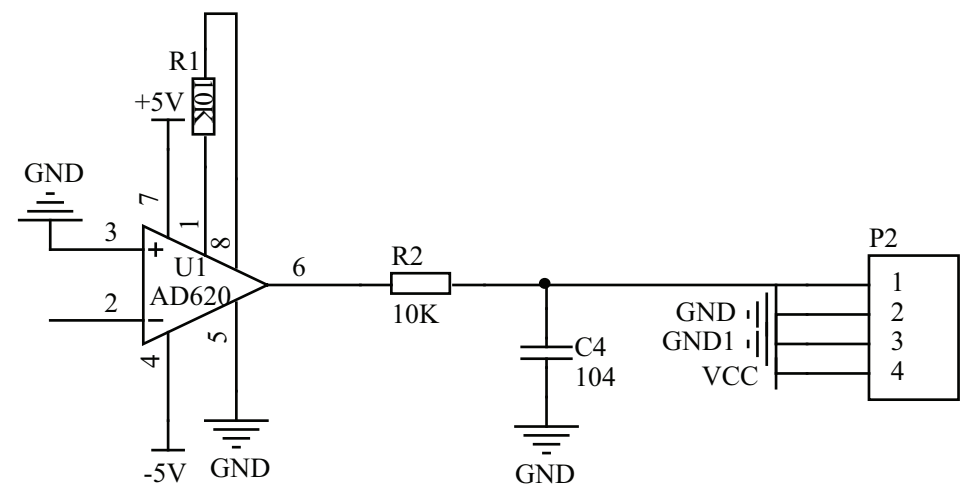

Fig. 4. Voltage amplifier circuit.

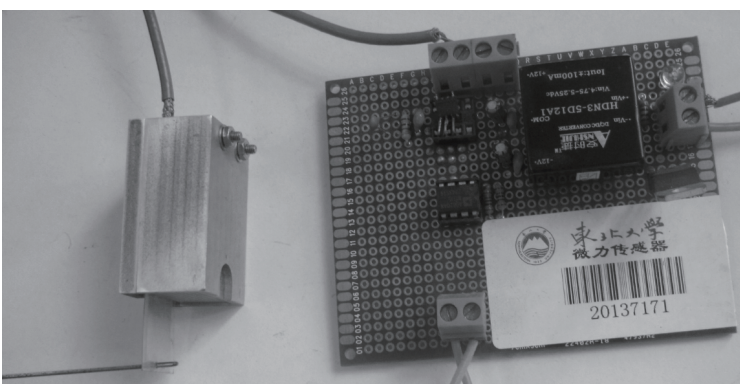

Fig. 5. Actual micro force sensor.

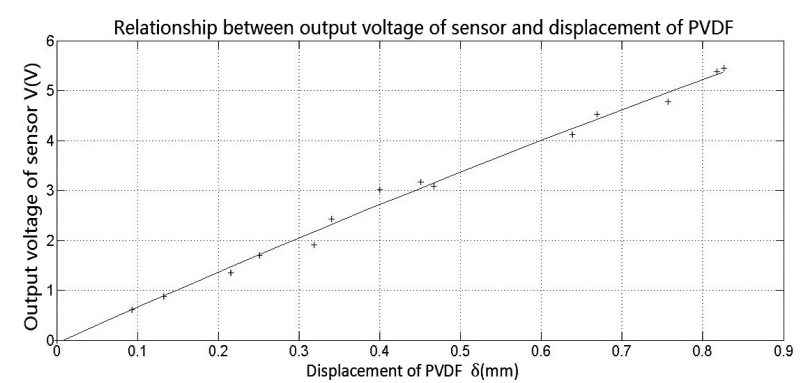

Fig. 6. The relationship between least square fitting output voltage and displacement.

\section{Experiment calibration}

\subsection{The relationship of the displacement produced by force and the generated voltage}

Through laser displacement sensor, the displacement of the PVDF generated by the force was measured. The conditioning circuit output perception signal was generated by the PVDF. Its experimental data was then analyzed using MATLAB software. The experimental data was fitted through the least square method. The relationship between the output voltage and the displacement in the PVDF is shown in the Fig. 6.

A fitted curve from the least square method was obtained as follow

$$
U=6.438 \delta-0.1987
$$

where $U$ represents the voltage which is generated by the signal conditioning circuit of the micro force sensor and $\delta$ represents the deformation of PVDF under the force.

\subsection{Calibration tests of the force and displacement}

The experimental facilitation is as shown in Fig. 3 and the displacement and stress of PVDF are calibrated. The stress of PVDF is calibrated through the electronic scale and the resolution ratio of the electronic scale is $0.01 \mathrm{~g}$. The fine tuning knob of micro operating floor is operated to adjust the 


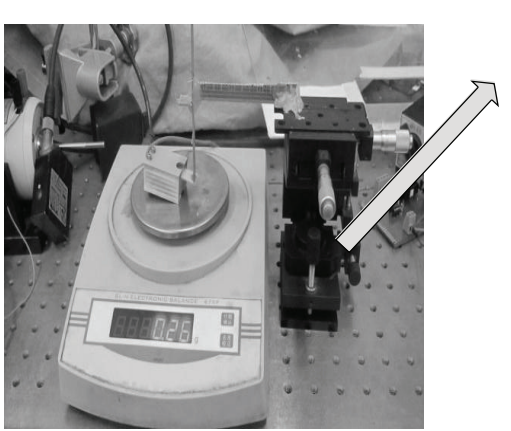

Fig. 7. The calibration experiment facility of displacement and stress of PVDF.
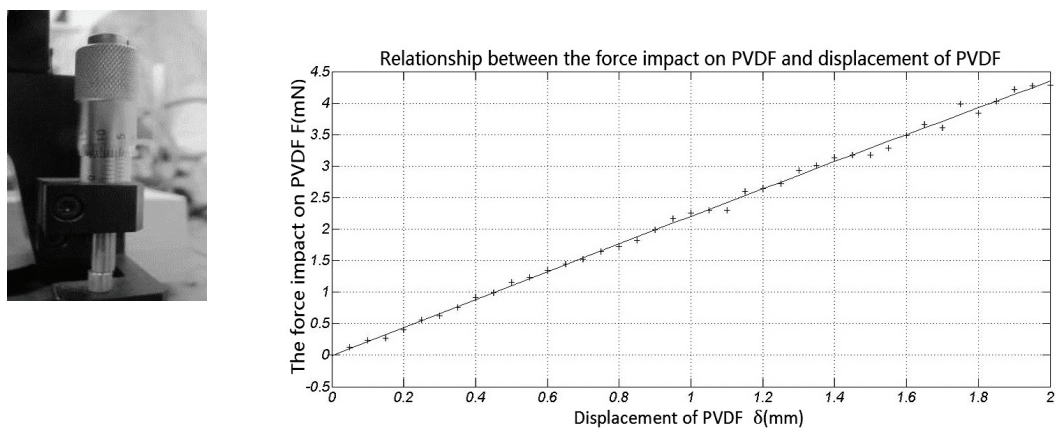

Fig. 8. The fitting curve of displacement and stress of PVDF.

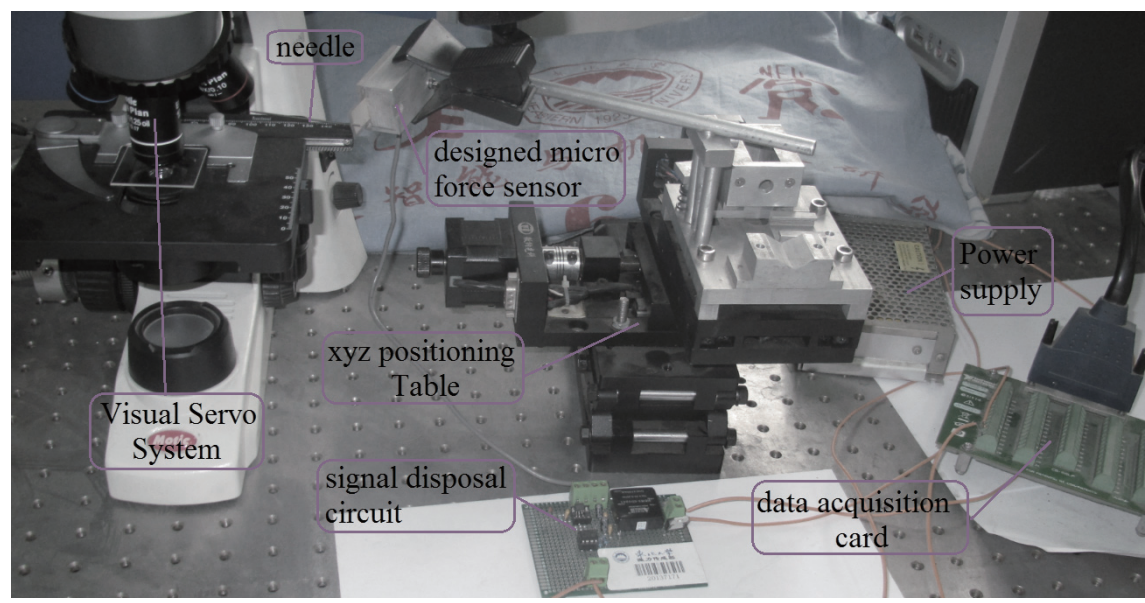

Fig. 9. Cell micro operations experiment platform.

displacement of the PVDF. The data recorded here is the one that was read out from the electronic scale. As noted, the experimental data was obtained from MATLAB using the least square method. Using the data, the experiment curve is shown in Fig. 8.

The least square fitting line is

$$
F=2.167842 \delta+0.0069
$$

where $F$ stands for the perceptive stress of the micro force sensor. $\delta$ stands for the deformation of the PVDF under the force. By combining Eqs (6) and (7) together, the formula for the relationship between output voltage and the stress of PVDF was obtained as

$$
F=\frac{1}{2.9793} U+0.06929
$$

where $U$ stands for the voltage from the signal conditioning circuit of the designed micro force sensor.

\section{Experimental results}

In Fig. 9, the system is the platform for cell micro operation. A carp egg was used in this bioexperiment stage. From Fig. 10(a), it can be seen that the micro-needle pierces into the membrane of the 


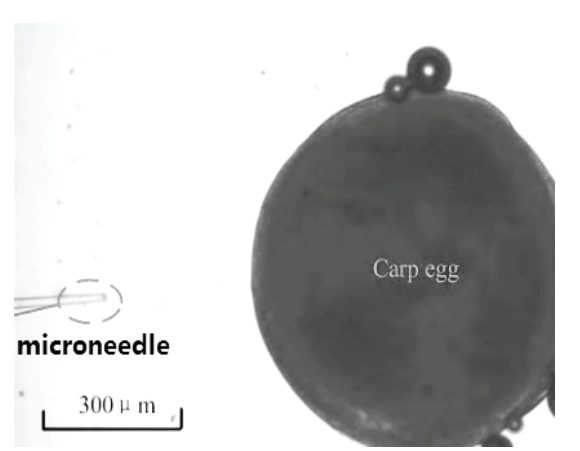

(a) The micro-needle pierce into the carp egg

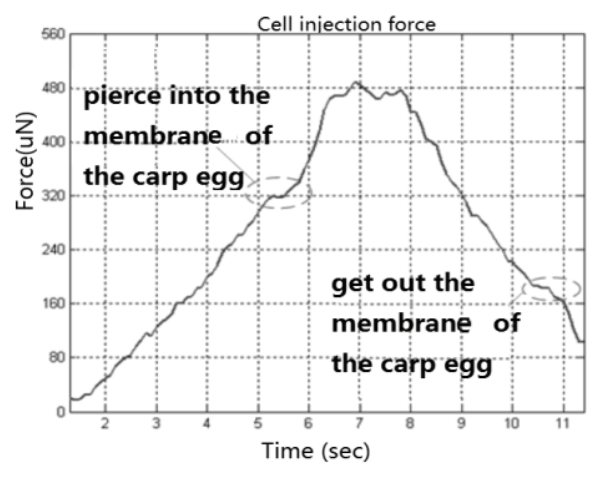

(b) The value of the force in the injection procedure

Fig. 10. Carp egg injection procedure.

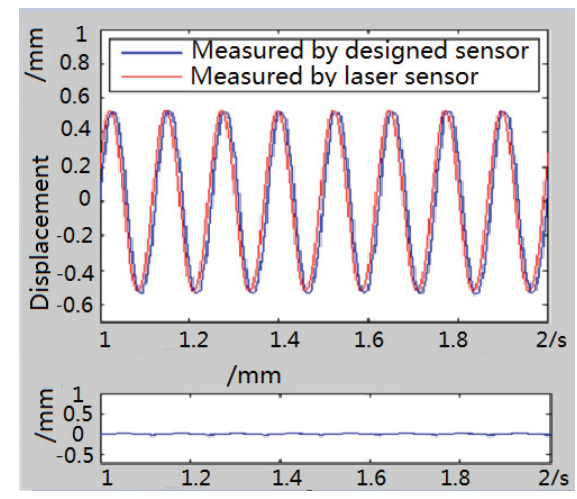

Fig. 11. Comparison of displacement.

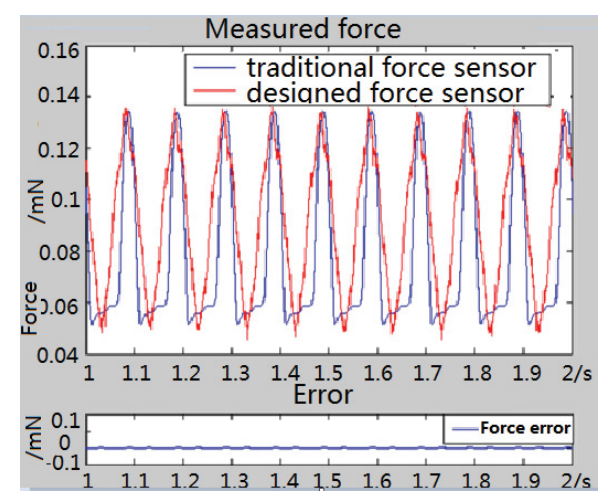

Fig. 12. Comparison of force.

carp egg. This is a microinjection process. In the process, the force of piercing into the membrane of the carp egg is approximately $480 \mu \mathrm{N}$. The force getting out from the membrane is approximately $320 \mu \mathrm{N}$.

The PVDF micro force sensor calibration was reasonable designed through the experimental tests. Firstly the voltage Eq. (1) of the relation for the stress and strain of the PVDF is derived as is. There is no consideration whether it is reasonable or not. Sinusoidal oscillations are then used to excite a sine wave signal of the PVDF. Through a laser displacement sensor, the motion of the IPMC displacement is thus detected. At the same time the data acquisition card is used to in order to collect the voltage signal. Using Eq. (6) the voltage will be converted to a corresponding displacement. This compares with the data which was measured through the laser displacement sensor. Using MATLAB, the curve is shown in Fig. 10. In the process of generating the voltage, error curves are also generated. The error curves show that the stress and strain of the calibrated PVDF are quite reasonable.

Secondly, through the experiment to detect sensing performance of the micro force sensor, a cyclical changing force was applied on the end of the mechanism for drive and sensing. The measured values of traditional force sensor were compared with the measured value of micro force sensor designed and presented in this paper. The measured values of the data curve drawings are shown in Fig. 12. The figure shows that the properties of drive and perception of the sensor designed and presented in this paper can perform more accurately than properties of drive and perception of the traditional force sensor. 


\section{Conclusion}

The sensitivity of PVDF micro force sensor designed in this paper is $\frac{d F}{d V}=3.334 \mu \mathrm{N} / \mathrm{V}$. The A/D converter samples at a resolution of a minimum voltage of $\frac{3 \mathrm{~V}}{2^{12}}$. Thus the minimum resolution is $2.44 \mu \mathrm{N}$. The linearity of the sensor is the ratio between the maximum deviation and the full scale $(10 \mathrm{mN})$. The maximum deviation between actual data and the fitting curve is $30 \mu \mathrm{N}$. Hence, the linearity of the micro force sensor is $\frac{30 \mathrm{uN}}{10 \mathrm{mN}}=0.3 \%$.

PVDF micro force sensors require the characteristic of high sensitivity in transformation between force and electricity. This characteristic was explored in the design of the micro force sensor presented in this paper. During the process of cell micro operation, the change of micro force between cell injections and cell membrane can effectively be detected and improved by this sensor. Hence, this can significantly improve the success rate of cell injection during micro operation.

\section{Acknowledgements}

This work was sponsored by the Chinese Defense Advance Research Program of Science and Technology, China under Grand No. 62501040412, Liaoning Baiqianwan Talents Program No. 2013921069, Science and Technology Foundation Project of Shenyang, No. F13-181-9-00, Science and Technology Foundation Project of Shenyang, No. F13-316-1-74, and Talent Resources Development Special Funds of Shenyang, No. 2010010403. The second author is supported by the program of the China Scholarships Council (No. 201406080054).

\section{References}

[1] H. Haibo, et al. A visual impedance force control of a robotic cell injection system. robotics and biomimetics, 2006. ROBIO '06. IEEE International Conference on. 2006.

[2] L. Chang, M. Howdyshell, W. Liao, C. Chiang, D. Gallego-Perez, Z. Yang, N. Muthusamy, L. Wu, L. J. Lee, R. Sooryakumar. Magnetic tweezer based 3D micro-electroporation system for high efficient cell manipulation and high throughput transfection. Small. 2014, DOI: 10.1002/smll201402564.

[3] K. Gao, L. Li, L. He, K. Hinkle, Y. Wu, J. Ma, L. Chang, X. Zhao, D. Gallego-Perez, S. Eckardt, J. Mclaughlin, B. Liu, D. F. Farson, L. J. Lee. Design of a microchannel-nanochannel-microchannel array based nanoelectroporation system for precise gene transfection. Small. 2013, DOI: 10.1002/smll.201300116.

[4] L. Chang, C. Liu, Y. He, H. Xiao, X. Cai. Small-volume solution current-time behavior study for application in reverse iontophoresis based non-invasive blood glucose monitoring. Sci China Chem. 2011, 54(1): 223-230.

[5] C. K. M. Fung, W. J. Li, I. Elhajj, N. Xi. Intemet-based remote sensing and manipulation in macro environment, 2001 IEEE IASME International Conference on Advanced Intelligent Mechatronics. 2001(2): 695700.

[6] C. K. M. Fung, I. Elhajj, W. J. Li, N. Xi. A 2-D PVDF force sensing system for micro-manipulation and micro-assembly. ICRA02. 2002, 1489-1494.

[7] I. Elhaj, Y. Shent, N. Xi, W. J. Li, J. Tan. A high sensitivity force sensor for microassembly: Design and experiments. Proceedings of the 2003 lEEE/ASME International Conference on Advanced Intelligent Mechatronics AIM. 2003, 703708.

[8] P. Brunetto, et al. A small scale viscometer based on an IPMC actuator and an IPMC sensor. Instrumentation and Measurement Technology Conference (I2MTC). 2010 IEEE. 2010.

[9] P. G. Datskos, T. Thundat, N. V. Lavrik. Micro and nanocantilever sensors in encyclopedia of nanoscience and nanotechnology [J]. H. S. Nalwa, Ed. American Scientific Publishers. 2004, X: 1-10.

[10] J. Sun, T. Liu. Developmentand application of piezoelectric polymer. China P Lastics Industry. 2005, 5(33). 